EstAg 30 (1995) 117-130

\title{
Sobre la Filosofía Analítica
}

Hablando en general, el análisis lingüístico en Filosofía comenzó a realizarse o bien apelando a la Lógica formal como proveedora de la forma y estructura de las sentencias clarificadoras -y debido a su neutralidad-, o bien parafraseando a base de expresiones que traduzcan y simplifiquen la complejidad inicial. El mayor 'handicap' está siempre en la equivalencia exacta de las transformaciones. A finales de la década de los años treinta empezó a aparecer en algunos un aire de desilusión respecto a estos tipos clásicos de análisis, pues la variedad y riqueza del lenguaje ordinario se resistían a ser encerradas en paráfrasis y traducciones; así surgió otra modalidad de análisis. La actividad analítica incide ahora sobre el lenguaje en el que se han expresado el científico, el moralista, el filósofo clásico, el hombre ordinario... Es más, puede decirse que a lo largo de la historia de este movimiento 'neoanalítico' se fue ampliando poøo a poco el análisis sobre áreas cada vez más amplias en discurso y temática filosóficas; en cualquiera de los casos la base fue un lenguaje sometido a reglas públicas en su funcionamiento, lo que garantizaba el estar moviéndose más allá de la propia subjetividad.

\section{El análisis clásico}

La corriente analítica se inició en Inglaterra a principios de este siglo como reacción al idealismo, concretado allí en las figuras de McTAGGART, F.H. BRADLEY... Los principales integrantes de esta oposición son G.E. MOORE y B. RUSSELL; ellos junto con el 'primer WITTGENSTEIN' han constituido la etapa clásica de la Filosofía Analítica.

G.E. MOORE propuso una lista de proposiciones (como "en el momento presente hay un cuerpo humano que es mío") que para él son totalmente obvias y dotadas de verdad en el sentido que tal palabra tiene en el uso ordinario, al mismo tiempo que tales enunciados expresan lo que ordinariamente expresan. Piensa que tienen un significado auténtico, el popular, y que, si alguien niega su verdad, del mismo hecho de negarla se sigue que ha tenido que sufrir algún error, ya que en realidad se niega a sí mismo y al mismo hecho de la negación. Así, lo que G.E. MOORE defendió, no fue el lenguaje 
común exactamente sino la cosmovisión del sentido común y su solidez, pero, eso sí, haciendo una reflexión constructiva sobre el lenguaje al clarificar el significado de varias afirmaciones, y al mostrar lo que un filósofo intenta decir, cuando dice lo que dice ${ }^{1}$.

B. RUSSELL llegó a la Filosofía por el afán de encontrar fundamento para confiar en la verdad de las Matemáticas, y, para ello, leyó a KANT y a HEGEL así como la Lógica de F.H. BRADLEY. Como resultado de las críticas de G.E. MOORE se opera en él un cambio desde posturas idealistas a otras más pluralistas y analíticas dentro de su ánimo de continuo perfeccionamiento. Pero B. RUSSELL desconfió del lenguaje ordinario y del sentido común, pues era consciente de que el mismo vocabulario ha influido sobre este último y nos ha inclinado a suponer que cada palabra se corresponde con un objeto. Para no dejarnos seducir por la morfología y sintaxis del lenguaje natural, buscó sustituirlo por otro exacto; por ello recurrió a un lenguaje ideal que pudiese captar la oculta forma Lógica de aquel, y bajo el supuesto de que los elementos básicos del lenguaje (las designaciones y predicados monádicos y poliádicos) se corresponden con los elementos últimos de la realidad (individuos, cualidades y relaciones). Creyó encontrar ese lenguaje ideal en la Lógica elemental de sus Principia Mathematica; ella habría de encargarse de dar un catálogo de los diversos géneros lógicos a los que habrían de ajustarse tanto las proposiciones como los hechos. Realizó este análisis y le sirvió de apoyo su "Teoría de las descripciones" como clarificadora de expresiones que, ostentando la calidad de sujetos gramaticales, aparentaban ser nombres genuinos ${ }^{2}$.

La otra gran figura de esta etapa clásica, y con una orientación 'formalista', es L. WITTGENSTEIN. Trabajó con B. RUSSELL desde su llegada a Cambridge en 1912 hasta su alistamiento en el ejército austríaco en 1914; el resultado de sus conversaciones será desarrollado por ambos con algunas diferencias, como las que aparecen entre las conferencias pronunciadas por B. RUSSELL sobre "Filosofía del Atomismo lógico" en Gordon Square (Londres, 1918) y las ideas expresadas por L. WITTGENSTEIN en el Tractatus Lógico-Philosophicus (publicado en 1921). Ambos evolucionaron por caminos distintos, pero va a predominar claramente la influencia de este último. Al igual que B. RUSSELL, piensa que el lenguaje ordinario disfraza el pensamiento, lo mismo que la forma externa del vestido puede impedir reco-

1. MOORE, G.E. : Some Main Problems of Philosophy. Allen and Unwin, London, 1953 (la ed. 1910)

2. RUSSELL, B. : "Filosofía del Atomismo Lógico" y "Atomismo Lógico" en Lógica y Conocimiento. Taurus, Madrid, 1966. 
nocer la forma del cuerpo; y esto es así por varias razones como la de que el hombre puede construir lenguajes sin tener idea de cómo y qué significa cada palabra. Es más, aunque las proposiciones del lenguaje ordinario estén ordenadas de un modo lógico, sucede como en una proyección geométrica en la que los rectángulos y las elipses se traducen en cuadrados y círculos; es decir, aunque sus proposiciones muestren propiedades lógicas, lo hacen de un modo confuso. Está convencido de que la esencia del lenguaje esta expresada en lo lógico, y de que su desconocimiento crea los problemas filosóficos. Esta "lógica" no es una experiencia, sino que es anterior a toda experiencia del "cómo", aunque no anterior al "qué". Las proposiciones lógicas "muestran" el armazón del mundo, o mejor, lo presentan y no "tratan" de nada ("sinnlos"); simplemente presuponen que los nombres tengan significado ("Bedeutung") y las proposiciones elementales, sentido ("Sinn"). Es decir, el lenguaje representa isomórficamente la realidad, y sin esa correspondencia no podría hablarse del mundo por medio del lenguaje. Esta "figuración" y correspondencia sólo pueden ser "mostradas", pero no "dichas", pues para esto habría que salirse al mismo tiempo del lenguaje. Con relación a ello hemos de limitarnos a significar lo indecible presentando lo decible, aunque al final haya que acabar por "tirar la escalera" una vez que se haya subido a ese nivel de esclarecimiento.

Estas convicciones le condujeron a un análisis en busca de una serie de proposiciones atómicas con unos signos simples, o nombres de objetos simples, relacionados entre sí de tal manera que sean "figura" -en el signo proposicional- de las relaciones entre esos objetos. Todas las proposiciones compuestas son funciones de verdad de proposiciones elementales por medio de constantes ógicas. El análisis es revelador de formas lógicas que guardan poca semejanza con las formas del lenguaje ordinario, en el que se usa una misma sintaxis lingüística para diferentes sintaxis lógicas.

No hay un campo de hechos para la Filosofía, por lo que tampoco tendrá un cuerpo de doctrina, quedándose en una elucidación de lo lógico. El filósofo no ha de ser un ciudadano de comunidad alguna de ideas; respondidas todas las cuestiones posibles científicas, no quedaría ya ninguna pregunta. La tarea de la Filosofía, en realidad, se limitará a esa actividad encaminada a aclarar proposiciones, descubriendo las proposiciones elementales sobre las que se apoya una proposición determinada; si el lenguaje fuese perfecto, la actividad filosófica seria inútil ${ }^{3}$.

3. WITTGENSTEIN, L.: Tractatus Lógico-Philosophicus. o.c. (Ver GARRIDO, M.: "La lógica del mundo" en VARIOS: Sobre el Tractatus Lógico-Philosophicus. Revista Teorema, número monográfico, 1972, p. 139-152 


\section{El análisis neopositivista}

Una etapa más en la Filosofía Analítica se debe a un conjunto de científicos, matemáticos y filósofos interesados en dar una fundamentación segura a la ciencia, y que estaban centrados en Viena; se consolidarán como "Círculo" a partir del manifiesto de 1929 y en sucesivos Congresos, hasta que a finales de la década de los años treinta llegó la dispersión de sus miembros, aunque no el fin del movimiento.

En su concepto de análisis hay que tener en cuenta, por una parte, las aportaciones que en el campo de las Matemáticas y de la Lógica venían haciéndose desde finales del siglo pasado, $y$, más próximamente, las doctrinas de B. RUSSELL y de L. WITTGENSTEIN en el Principia Mathematica y en el Tractatus respectivamente. Este último libro será visto como un apoyo a la Lógica e, incluso, a lo empírico por hablar de "simples" y de hechos cuya expresión en proposiciones elementales haría desaparecer la arbitrariedad en el simbolismo con el que describimos los hechos del mundo. Por otra parte, está la importancia que dan a la verificabilidad por medio de lo observacional para determinar el sentido de una proposición. Esto es tanto como conocer las condiciones de verdad de una proposición en cuanto a la empiricidad de sus términos, o a la decidibilidad sobre su verdad o falsedad con predicados observables, o a su pertenencia a una clase finita y lógicamente consistente de oraciones observacionales (según C.G. HEMPEL); la verificación, por su parte, se encargará de proporcionarnos su verdad. La fijación de tal verificabilidad ha sido muy variada. Se ha ido desde una formulación fuerte exigiendo la constatación directa y personal y la traducción a un lenguaje fisicalista, hasta otras más débiles como la posibilidad de verificación a la espera de medios técnicos, o el contar con observaciones relevantes de algunas premisas, o el ver que la proposición es construible por tener la forma de hechos ya ocurridos.

Bajo estos principios los dos únicos tipos significativos de enunciados fueron los "fácticos" y los "formales". Los primeros son acerca de hechos y en ellos se puede hablar de verificabilidad y de verificación; realmente están apuntando hacia una fundamentación en unos enunciados elementales ("protocolares" para O. NEURATH, "primarios" para R. CARNAP, u "observacionales" para C.G. HEMPEL) en forma de constataciones básicas. Los segundos no dicen nada de los objetos de experiencia, y basta en ellos la coherencia y la compatibilidad con el resto para hablar de verdad (formal).

Con todo ello el campo abarcado por la Filosofía quedaba terriblemente limitado. Según M. SCHLICK en "El futuro de la Filosofía" ${ }^{4}$ sus cuestiones

4. SCHLICK, M.: "El futuro de la Filosofía" en MUGUERZA, J.: La concepción analítica de la Filosofía. Alianza, Madrid, 1974, p. 291. 
o bien desaparecían como tales al demostrarse que eran malentendidos lingüísticos, o bien se descubría que no eran sino cuestiones científicas ordinarias disfrazadas. Y si las proposiciones de la ciencia agotan todo lo que puede ser dicho con sentido por eso de que expresabilidad y verificación son una y la misma cosa, es fácil imaginar su interés en la descalificación de la Metafísica; esta labor la realizan, sobre todo, desde el análisis lógico del lenguaje -por ejemplo, O. NEURATH y, más aún, R. CARNAP ${ }^{5}$, ya que desde el análisis semántico no les resultaba tan fácil y cómodo, aunque el origen de todo estuviera en la falta de significado de estas proposiciones al no salvarse en ellas el principio de verificabilidad. La labor Analítica de la Filosofía se redujo a eliminar palabras asignificativas y proposiciones carentes de sentido, a hacer sintaxis Lógica del lenguaje científico, a precisar el significado de los enunciados científicos, a la traducción de un lenguaje a otro dentro del conjunto lingüístico de la ciencia...

Esta radicalización fisicalista, antimetáfisica y restriccionista se produjo básicamente en el período de 'entre-guerras', dándose después una apertura a la temática filosófica. Un buen ejemplo es R. CARNAP cuyo radicalismo se mitigaría según nos acercamos a la década de los años cincuenta (quizás por influjo de A. TARSKI), a medida que se va convenciendo de que sus interpretaciones del Tractatus y su actitud hacia la Filosofía eran demasiado empobrecederas; lo semántico era irreductible, aunque su tratamiento no deba de sobrepasar los límites internos de un determinado lenguaje, por lo demás, elegido por conveniencias pragmáticas. Esta apertura vino precedida por una reacción y crítica a sus principios y doctrinas. Es ya un tópico el hablar de la inverificabilidad de la proposición que enuncia el criterio; pero las críticas se extendieron a otras doctrinas y con variedad de aspectos. Tal es el caso de la inverificabilidad de las proposiciones generales, o el de la dificultad de precisar las experiencias básicas sobre las que fundamentar las proposiciones elementales sin caer en el solipsismo o en fenomenalismos, o el de la obligación de recurrir en sus descripciones científicas a teorías que contienen conceptos no empíricos elaborados para ordenar la experiencia... A pesar de lo efectiva que pudo ser la crítica, lo cierto es que este movimien-

5. SCHLICK, M.: "Positivismo y Realismo" y "Sobre el fundamento del conocimiento" en AYER, A.J.: El positivismo lógico. F.C.E., México, 1965, pp. 88-114 y 215-232 respectivamente.

NEURATH, O. : "Proposiciones protocolares" en AYER, A.J.: El positivismo lógico. o.c., p.205-214.

CARNAP, R. : "La antigua y la nueva lógica" y "La superación de la Metafísica mediante el análisis lógico del lenguaje" en AYER, A.J. : El positivismo lógico. o.c., pp. 139-152 y 66-87 respectivamente.

“ ": "Filosofía y Sintaxis lógica" y "Empirismo, Semántica y Ontología" en MUGUERZA, J. : La concepción analítica de la Filosofía. o.c., pp. 294-337 y 400-419 respectivamente. 
to siguió dándose en diversas latitudes -sobre todo, en Estados Unidos de América- y de muy variadas formas bajo modalidades renovadoras. También hay que dar por admitido el estímulo que el análisis neopositivista ha supuesto para un sector de investigaciones filosóficas en las últimas décadas, sobre todo, en lo relativo a la fundamentación y límites del conocimiento humano a tenor del modelo científico y del instrumento lógico.

\section{El análisis en el 'último' WITTGENSTEIN}

Desde su vuelta a Cambridge a principios de 1929 se observa en su doctrina una reconsideración de la relación lenguaje-lógica-realidad junto a un abandono de la imagen supersensible del lenguaje, lo cual le lleva a fijarse en la multiplicidad de clases de palabras y de sentencias, y en la diversidad de funciones a pesar de una aparente uniformidad. Se queda atrás la doctrina de los elementos simples de la realidad conexionados con nombres auténticos de un lenguaje, pues no está de acuerdo con que el significado de una palabra sea el objeto al que se aplica. Esa es una concepción del lenguaje que se enmarca en las formas primitivas de éste. Es más, con ese lenguaje "nominativo" no se conocería el significado de muchas palabras como las series numerales o el de otras tan corrientes como "abajo", "aquí"... Frente a la Teoría del Tractatus WITTGENSTEIN repetirá que el significado de una proposición es su "uso" o "empleo". Piensa que antes estaba bajo la ilusión de que en la Lógica, debido a su pureza, exactitud y generalidad, se tiene la clave para encontrar la esencia y la clarificación del pensamiento, del lenguaje e, incluso, del orden de posibilidades comunes al mundo y al pensamiento.

Esta ilusión desaparece, si se renuncia a buscar la esencia del lenguaje y se examina lo que en todo tiempo está ante nosotros: el funcionar actual del lenguaje. Cuando hablamos del lenguaje como de un simbolismo en un cálculo exacto, sólo en casos muy contados, como el de las Matemáticas y el de las Ciencias, podemos encontrar aquello en lo que estamos pensando ${ }^{6}$.

Las actividades lingüísticas son tan variadas como los juegos; es más, son "juegos", pues, como ellos, hay una regularidad de uso presidiendo el modo de operar de las expresiones. Estas reglas, aunque no tienen la convencionalidad de las del juego al imponérsenos por estar inmersos en el lenguaje, tampoco agotan la capacidad creadora al emplear expresiones, por lo que, más que normas, son cauces posibilitando la originalidad en la conducta lin-

6. WITTGENSTEIN, L.: Philosophical Investigations.. o.c., parte I, núms. 91, 95, 96, 97, 98. 
güística ${ }^{7}$. Ahora bien, cuando se fuerza el lenguaje con pretensiones de profundidad y ultimidad a base de formas distanciadas de las ordinarias, se llega a situaciones que producen "enredo" (espasmo, calambre, niebla) mental. Esa confusión y desconcierto procedentes de usos "desconcertantes" de expresiones que nos inclinan a pensar que ahí hay cosas extrañas ocultas, es la causa de nuestros problemas filosóficos; ésta es la falaz tentación "esencialista”. Así, gramáticas aparentemente análogas nos llevan a pensar en actividades análogas; el ansia de generalidad nos lleva a pensar que tiene que haber algo común, como propiedad, a todas las cosas incluidas bajo un nombre general, el cual sería el significado de la palabra. No nos damos cuenta de que la relación de los términos a las cosas se reduce a una actividad bajo ciertas reglas que posibilitan multiples usos. El filósofo maneja otro lenguaje en el que, por haber introducido cambios en los usos del lenguaje ordinario, surgen problemas que no surgirían en éste ${ }^{8}$.

El concepto de análisis en este 'último' WITTGENSTEIN se corresponde con las ideas anteriores. No se trata de explicar ni de deducir nada, sino de dejarlo todo como está; no se encamina a captar algo nuevo, sino hacia lo ya visto y sabido de todos pero que ahora hay que entender, es decir, hacia algo que sabemos cuando nadie nos pregunta. El objetivo esta en trazar los límites del pensamiento estableciendo los límites del lenguaje. Ya no se trata de un análisis por descomposición del lenguaje en sus elementos últimos, sino de un análisis por comparación de juegos lingüísticos a través de similitudes y diferencias. Nuestras consideraciones se ocuparan de describir lenguajes, de ver la gramática de diversas expresiones parecidas a los términos generales, de atender a casos particulares en los que se usan las palabras, de precisar "en qué circunstancias decimos...", de recoger el conjunto de semejanzas que se superponen y entrelazan constituyendo el aire de familia de los diferentes usos de una palabra... La dificultad es como un caso en el que nos parece que nos faltan o nos sobran piezas de un rompecabezas; pero ahí están todas, sólo que revueltas, sin que sirva de nada aplicar la fuerza para hacerlas encajar, sino que lo que hay que hacer es observarlas cuidadosamente y ordenarlas 9 .

Por una parte, es innegable que para L. WITTGENSTEIN, al no haber en origen auténticos problemas sino enredos y malos usos, tampoco habrá

7. “ “: Philosophical Investigations. o.c., parte I, núm. 66.

“ ": Cuadernos azul y marrón. o.c., pp. 54.

8. " ": Cuadernos azul y marrón. o.c., pp. 27, 34, 46.

9. “ ": Cuadernos azul y marrón. o.c., pp. 43, 46, 47, 48, 78, 89, 95, 212

“ ": Philosophical Investigations. parte I, núms. 90, 109, $116,124,126$ 
auténticas soluciones, sino disoluciones, y que el tratamiento de una cuestión será parecido al de una enfermedad; igualmente habrá muchos métodos filosóficos lo mismo que hay muchas y diferentes terapias. Pero, por otra parte, parece que no todo quedaba reducido a deshacer ambigüedades y a quitar golpes en la cabeza por chocar contra los límites del lenguaje; dado que una expresión puede resultar significativa, si es usada con propósito comunicativo en un contexto dado del lenguaje, después de limpiar el campo filosófico y de eliminar falsos usos, cabía la posibilidad de construir edificios nuevos y de preguntar ya en forma correcta por nuevas cuestiones. Exactamente esto es lo que se hizo tan pronto como se conocieron los últimos escritos de L. WITTGENSTEIN, y se impuso su nueva alternativa al análisis valorando el lenguaje ordinario en detrimento del reduccionismo lógico-formal. Si bien el lenguaje científico se presta a la exactitud de lo lógico, en la intercomunicación humana hay más misiones a cumplir y de ellas responde el lenguaje ordinario. Este es de suyo no sólo significativo sino que lo es de diversos modos según los diversos usos. Así nació la etapa más larga y última de la Filosofía Analítica: la "Filosofía del Lenguaje Ordinario".

\section{El análisis terapéutico}

En la Universidad de Cambridge se continuó el nuevo análisis entre los mismos discípulos de L. WITTGENSTEIN a impulsos de sus lecciones y a la vista de sus notas de clase, aunque se contaba allí con el antecedente de otro pensador interesado en el lenguaje ordinario, G. MOORE. Este grupo cantabrigense de analíticos no fue numeroso, ni tampoco duró muchos años; ello puede explicarse en parte por la atracción que ejerció Oxford tan pronto como llegaron allí estas nuevas doctrinas, al ser un centro en el que se daba más importancia académica a saberes de carácter humanístico.

El tipo de análisis que allí se hizo, fue diseñado por J. WISDOM, sucesor de L. WITTGENSTEIN en su cátedra (1952). Sus doctrinas anteriores habían pasado por el reduccionismo lógico-formal al que acabó desechando como teoría metafísica, y por el neopositivismo al que acabó considerando dogmático. Desde mediados de los años treinta y a lo largo de un buen tiempo fue básicamente a través de sus artículos el modo como se pensó saber sobre la evolución del pensamiento de L. WITTGENSTEIN, ya que no se publicaba nada de este desde el Tractatus. Ahora, siguiendo tanto a éste cuando hablaba de que los problemas filosóficos son algo similar a calambres mentales con matizaciones enfermizas, como a G. MOORE que presentaba soluciones a las confusiones de los filósofos idealistas, la Filosofía adquirió una concepción paradójica, y el análisis, un sentido terapéutico. 
J. WISDOM habló de que el problema especulativo expresa una perplejidad, y de que se trata de paradojas y vulgaridades debidas al mal uso del lenguaje. El metafísico hace sugerencias descabelladas en las que, lo que habría sido valido para nosotros, pasa a ser algo vacilante. Estas paradojas metafísicas han de ser compensadas por la re-aserción de antiguas verdades opuestas a ellas, lo mismo que nos liberamos de una paradoja psicológica. Quizás lo más típico de J. WISDOM sea el querer ver en lo metafísico una contraverdad iluminadora, un uso desconcertante del lenguaje que en el mejor de los casos puede llamar la atención sobre diferencias y semejanzas lingüísticas de las que no caemos en la cuenta en la vida ordinaria ${ }^{10}$.

Cabia radicalizar esta orientación, y así sucedió cuando la nueva Filosofía Analítica prosperó en U.S.A. por influjo tanto del mismo L. WITTGENSTEIN como de J. WISDOM; tal es el caso de Morris LAZEROWITZ. Ya J. WISDOM había comparado la Metafísica con el Psicoanálisis apoyándose en que ambos descubren problemas latentes, en que ambos tienen un carácter pedagógico y sorprendente en sus enunciados, en que la perplejidad y la paradoja son como una neurosis que se cura con la clarificación de lo aparentemente contradictorio... Ahora se llegó más lejos y se habló de fantasías gratificantes sustitutivas de algún deseo irrenunciable, o de frustraciones internas por desconocimiento de su inconsciente y que se proyectan al exterior en forma de esencias y naturalezas ${ }^{11}$.

La manera de entender el análisis J. WISDOM se ensanchó con F. WAISMANN, quien de 'lector' en Cambridge pasó a Oxford como Profesor de Filosofía de las Matemáticas y de las Ciencias. Para él la Filosofía, además de hacer análisis crítico del lenguaje, de detectar ofuscaciones y de clarificar el sentido de las expresiones, tiene una misión que cumplir aportando nuevas visiones y aspectos más amplios de la realidad; es más, para él era un sinsentido decir que lo metafísico carece de sentido ${ }^{12}$.

\section{El análisis oxoniense}

Aunque fue Cambridge el primer lugar de la "Filosofía del Lenguaje Ordinario", ya que allí profesó L. WITTGENSTEIN, fue Oxford el lugar donde esta Filosofía se cultivó por más tiempo y por más número de filóso-

10. WISDOM, J. : "Filosofía, Metafísica y Psicoanálisis" en MUGUERZA, J. : La concepción analítica de la Filosofía. o.c., p. 420454.

11. LAZEROWITZ, M. : The Structure of Metaphysics. London-New York, 1955.

“" : Philosophy and Illusion. London-New York, 1968.

12. WAISMANN, F.: "Mi visión de la Filosofía" en MUGUERZA, J. : La concepción analítica de la Filosofía. o.c., p. 491-528. 
fos, y donde se desarrollaron en versiones peculiares las posibilidades que había en la doctrina wittgensteiniana. Dentro de un sistema de estudios basado en el saber clásico las ideas del 'segundo' WITTGENSTEIN respiraron una nueva atmósfera y se gestó una concepción más ampliada y positiva. Es decir, al mismo tiempo que se mantiene la investigación filosófica en los usos ordinarios de las palabras y de las expresiones del lenguaje natural, se debilita la tesis de que los problemas filosóficos sean simples errores de uso, y la de que la tarea del filósofo sea el diagnóstico, cura y rehabilitación de los abusos lingüísticos; así se fortalecen y consolidan ideas más constructivas. Buena parte de sus doctrinas se presentaron en forma de artículos (en Mind, en The Philosophical Review...) o de ponencias (publicadas después en los Proceeding como los de la "Aristotelian Society"). Con ello la "Oxford's School" se convirtió en el foco más representativo de la Filosofía Analítica desde comienzos de los años cincuenta; bastó muy poco tiempo, una década, para que la nueva corriente se consolidase como tal y se extendiese en el espacio y en el tratamiento de temática.

La verdad es que no se hicieron 'manifiestos', ni se puede hablar de 'una' doctrina específica propia, pero se compartieron ciertas actitudes como es la preocupación por la elucidación de conceptos, y las actividades metodológicas basadas en el lenguaje. Se cayó en la cuenta de que el universo conceptual es inseparable del sistema lingüístico, y de que las confusiones lingüísticas están reflejando errores conceptuales. No se trató de conocer estructuras internas del lenguaje, sino de captar el significado real de conceptos y de proposiciones en sus usos ordinarios actuales y vivos. Es más, hay que ocuparse no sólo de nuestros conceptos sino también de sus relaciones e, incluso, de la estructura de nuestro sistema conceptual, si bien tendrá que ser a través del lenguaje ordinario; junto al rechazo de una Filosofía limitada a la sintaxis lógica se potenció el estudio de aspectos semánticos del lenguaje, llegando a la investigación concreta y minuciosa de las diversas situaciones en las que se emplean las expresiones. Tal es el caso de G. RYLE y de J. L. AUSTIN, que hacen de pioneros ${ }^{13}$.

En cuanto a expansión, las nuevas ideas se difundieron principalmente por paises de habla inglesa, sobre todo, U.S.A., donde ya existían las otras

13. RYLE, G. : The concept of mind. Hutchinson, London, 1949.

“" : Dilemmas. Cambridge Univ. Press, Cambridge, 1954.

“ " : Collected Papers. (2 vols.). Hutchinson, London, 1971.

AUSTIN, J.L.: Philosophical Papers. Oxford Univ. Press, London, 1961; trad. en Rev. de Occidente, Madrid, 1975.

" ": How to do things with words. Harvard Univ. Press, Cambridge (Mass.), 1962; trad. en Paidos, Buenos Aires, 1971 
corrientes analíticas, como la neopositivista y la terapéutica. También se propagó algo por las Europa continental y muy poco en Hispanoamérica.

Respecto a la ampliación de temática, se hicieron aportaciones doctrinales en diversos campos filosóficos entre los que están los siguientes:

- En Metafísica quedaron atrás doctrinas wittgensteinianas como la de que, cuando alguien quiera hacer Metafísica, se le tendría que mostrar que no había dado significado correcto a ciertas expresiones, o la de que respecto a lo indecible lo mejor es callarse. Por una parte, la evolución desde el 'segundo' WITTGENSTEIN y, por otra, las reiteradas críticas al neopositivismo tanto por su fundamentación "dogmática" como por su cambiante criterio de verificabilidad posibilitaron esta apertura en la palabra "significado" y con ello la significatividad de este tipo de discurso. El resultado fue la aparición de varios proyectos y realizaciones, sobre todo, en la década de los sesenta ${ }^{14}$.

- Otra materia de estudio por la que estos filósofos mostraron predilección, fue la Etica como si se tratase de empalmar con la vieja inquietud práctica del empirismo inglés, al mismo tiempo que se hacia un intento por superar el emotivismo ético imperante en la doctrina de corte neopositivista. Como es de suponer, se hace teoría ética mediante el análisis de las expresiones morales del discurso ordinario abordando un amplio repertorio de cuestiones, como la del valor de la libertad y decisión personal en las actuaciones humanas, la del significado de los términos usados en el lenguaje ético, la de la distinción entre enunciados descriptivos y preceptivos, la de la relación entre "ser" y "deber", la de la validez lógica de la argumentación moral, la de la posible fundamentación de los principios morales... Esto condujo a la aparición de un amplio numero de libros y artículos desde mediados de los años cincuenta hasta el comienzo de los setenta ${ }^{15}$.

- Otro sector filosófico tratado fue el educacional. La verdad es que ya venía haciéndose desde bastante antes y fuera de Inglaterra, concretamente en U.S.A. y desde la llegada de los pensadores del "Círculo de Viena" al disolverse éste en la Europa continental por causa de la perse-

14. HARE, R.M.: Essays on Philosophical Method. Macmillan, London, 1972, p. 38-53.

TOLMIN, E.W.F. : "Mr. Hare's paper: a rejoinder", Ratio, 1960, 3, núm. 1, p. 1-8.

HAMPSHIRE, S.N. : "Metaphysical systems" en PEARS, D.F. (Ed.) : The Nature of Metaphysics. Macmillan, London, 1965, p. 23-38. 1959.

STRAWSON, P.F. : Individuals. An essay on Descriptive Metaphysics. Methuen, London,

15. Ver MUGUERZA, J.: La razón sin esperanza. Taurus, Madrid, 1977. 
cución nazi. Esto se vio potenciado por la necesidad que sociedad, políticos y pensadores norteamericanos sentían de encontrar una alternativa al paradigma liberal-experimentalista-progresista de J. DEWEY. El capítulo dedicado a la Filosofía educacional analítica en el LIV YEAR$B O O K$ de la 'National Society for Study of Education' (1955) y el monográfico de la Harvard Educational Review (1956), así como la consolidación del análisis filosófico en Inglaterra, hicieron que esta temática y bajo el paradigma analítico alcanzase un fuerte desarrollo en el mundo de habla inglesa durante los años sesenta hasta el punto de que apenas tuvo críticas; esto último sucedió al comienzo de la década siguiente. En aquellos años todos parecían coincidir en que era fundamental la clarificación de un lenguaje confuso como el empleado por las Ciencias de la Educación, y en que la solución estaba en el análisis del lenguaje ordinario; al mismo tiempo se disiparían problemas y enredos procedentes de usos indebidos ${ }^{16}$.

- Aún cabe hablar de otras cuestiones por las que los "analíticos" mostraron interés; por ejemplo, el de la metodología de las ciencias sociales e históricas. La explicación causalista de la conducta humana vigente en las concreciones neopositivistas a base de leyes generales o de estadísticas al modo del mundo de la Física se vio enfrentada a una interpretación teleológica que ya se empezó a dibujar a partir del 'segundo' WITTGENSTEIN, cuando el término "causa" fue sustituido por el de "acción" y comenzaron a tenerse en cuenta la intención, el propósito y la finalidad. Este fue otro campo en el que durante esos mismos años aparecieron varios libros y artículos.

Una consideración final respecto a la Filosofía Española de estos últimos años

La Filosofía Analítica fue uno de los movimientos que llegaron a nuestro país en la década de los años sesenta junto con el dialéctico y el fenomenológico; también en su compañía supo jugar su papel frente a la Filosofía institucionalizada. No se trata de asignar todo el trabajo de renovación del ambiente filosófico español a estos movimientos. La verdad es que hay que

16. Son muchos los filósofos que han desarrollado este campo. Entre ellos están: ARCHAMBAULT, R.D., DEARDEN, R.F., HIRST, P.H., O'CONNOR, D., PETERS, R.S., SOLTIS, J.F. Libros representativos pueden ser:

ARCHAMBAULT, R.D. (Ed.) : Philosophical analysis and education. Routledge and Kegan Paul, London, 1965

PETERS, R.S. : Education as Initiation. Univ. of London, London, 1966

“ “(Ed.) : The Philosophy of Education. Oxfor Univ. Press, London, 1973. 
citar -aunque sea de pasada- a otros factores que venían facilitando el camino. Me refiero a un cierto liberalismo intelectual que comenzaría con la revista Escorial agrupando a D. RIDRUEJO, P. LAIN ENTRALGO y A. TOVAR, y que se ampliaría después a pensadores tanto de inspiración orteguiana como de orientación espiritualista cristiana (X. ZUBIRI, J. MARIAS, J. ROF CARBALLO...). ahí están también las aportaciones críticas de temática sociopolítica de E. TIERNO GALVAN -además de su traducción del Tractatus en 1954- y las de moral de J. L. ARANGUREN, o las de la revista Teoría (1953-1954) sobre Filosofía de la Ciencia -dirigida por C. PARIS y M. SANCHEZ MAZAS- ...

El caso es que al llegar los años sesenta empezaron a aparecer publicaciones en las que se dialoga aquí en España con otros movimientos filosóficos contemporáneos entre los que se encuentra la Filosofía Analítica; éstas tenían procedencias tan diversas como C. PARIS, F. MONTERO MOLINER, J. GOMEZ CAFFARENA, E. LLEDO... En buena parte se la asoció con el cultivo de la Lógica formal, y ello tenía como base no sólo el que la Lógica fundamentaba un tipo de análisis, sino también la aparición de libros como los de M. SACRISTAN, A. DEAÑO, J. MOSTERIN... Pero sus resultados también se dieron en los campos del discurso científico y del moral, sobre todo. Ahí están las contribuciones de J. HIERRO con el primer libro de Filosofía Analítica sobre tema moral publicado en España (Problemas del análisis del lenguaje moral, 1970); los prólogos a las traducciones de G. FREGE de J. MOSTERIN; las traducciones aparecidas en la Colección "Estructura y función" de la Ed. Tecnos; los trabajos de J. MUGUERZA con sus facetas tanto críticas como 'autocríticas'; la labor de las revistas Teorema -dirigida por M. GARRIDO- , Crítica - dirigida por A. ROSSI y otrosy Anuario filosófico. -llevada por J. GARCIA LOPEZ y J. CRUZ CRUZ-; las Indagaciones sobre el lenguaje (1970), Las palabras y las cosas (1971) y El cambio de marcha en Filosofía (1974) del recientemente fallecido J. FERRATER MORA; el tratamiento de las diversas modalidades de análisis en Lenguaje, Filosofía y Conocimiento (1973) de J. L. BLASCO, o La estructura del mundo sensible (1973) de U. MOULINES...

Todo parecía asegurar un tiempo de vida y un cierto lugar a la Filosofía Analítica en los ambientes filosóficos españoles, y no faltó quien así se expresó; era el momento en el que estaba abierto el debate entre "analíticos" y "dialécticos" como las dos líneas que tenían mayor audiencia entre los "filósofos jóvenes" españoles; a veces entre ambas líneas no había una separación tan tajante, pues en el fondo compartían inquietudes e, incluso, temática. 
Quizás debido a que se limitó demasiado el campo de la reflexión analítica al reducirlo casi sólo a la epistemología, a la ética y algo a lo educacional, con lo que faltó capacidad para llevar el análisis a otras cuestiones y sectores típicamente filosóficos, o quizás debido a que aquello que se venía haciendo pecaba a veces de "insustantivo" como si la Filosofía tuviese sólo una función "adjetiva" y secundaria, el caso es que poco a poco se impuso la tesis de que había que ir más lejos y por otros cauces, y sobre el análisis cayó, cuando menos, el silencio y, cuando más, la acusación de "plomizo", "insulso" y "aburrido", o la descalificación como auténtica "eutanasia" de la Filosofía. La despedida tuvo mucho de "portazo".

Ante esta situación y con la tranquilidad que proporciona el ambiente de silencio, no estará de más el mirar un poco hacia atrás, el estudiar algunas doctrinas de esos momentos y concreciones del movimiento analítico, el conocer un poco mejor algo que por aquí pasó deprisa y que en países de habla inglesa ocupó algo más de medio siglo ${ }^{17}$. No es resucitar muertos, sino simplemente hacer Historia y juzgar con la visión de conjunto que da cierta distancia en el tiempo, y por eso de que puede haber algo a tener en cuenta; su estudio quizás pueda servir para 'limpiar' y 'afinar' lenguajes y 'discursos', para precisar conceptos, para plantear con más exactitud cuestiones. ¿Quién tiene razón? ¿La descalificación total? ¿Posturas intermedias como la de J. FERRATER MORA, cuando decía que "la Filosofía analítica no sobra. Pero tampoco basta"? ${ }^{18}$. Si esto último fuese conceder demasiado, al menos merecería la pena el aprobar una asignatura pendiente relativa a la clarificación crítica de posibles aportaciones por parte de un movimiento filosófico en un siglo como este tan ocupado en el estudio del lenguaje desde campos y perspectivas tan distintos.

Juan-Tomás PASTOR GARCIA Universidad de Valladolid

17. Debo hacer alusión al libro de Wenceslao J, GONZALEZ FERNANDEZ : La Teoría de la Referencia. o.c. $119-120$

18. FERRATER MORA, J. : Cambio de marcha en Filosofía. Alianza, Madrid, 1974, p. 